\title{
Pharmacokinetic Parameter Date Time of Reference Timepoint
}

National Cancer Institute

\section{Source}

National Cancer Institute. Pharmacokinetic Parameter Date Time of Reference

Timepoint. NCl Thesaurus. Code C87979.

The date and time of a pharmacokinetic parameter reference point. 